\title{
Costeo basado en actividades: una metodología de gestión en tratamientos intensivos
}

\author{
SANDRA ALVEAR V. ${ }^{1, a}$, JORGE CANTEROS G. ${ }^{2}$, \\ JUAN JARA M. ${ }^{3}$, PATRICIA RODRÍGUEZ C. ${ }^{\text {,a }}$
}

\section{Activity-based costing methodology to manage resources in intensive care units}

Background: An accurate estimation of resources use by individual patients is crucial in hospital management. Aim: To measure financial costs of health care actions in intensive care units of two public regional hospitals in Chile. Material and Methods: Prospective follow up of 716 patients admitted to two intensive care units during 2011. The financial costs of health care activities was calculated using the Activity-Based Costing methodology. The main activities recorded were procedures and treatments, monitoring, response to patient needs, patient maintenance and coordination. Results: Activity-Based Costs, including human resources and assorted indirect costs correspond to 81 to $88 \%$ of costs per disease in one hospital and 69 to $80 \%$ in the other. The costs associated to procedures and treatments are the most significant and are approximately $\$ 100,000$ (Chilean pesos) per day of hospitalization. The second most significant cost corresponds to coordination activities, which fluctuates between $\$ 86,000$ and 122,000 (Chilean pesos). Conclusions: There are significant differences in resources use between the two hospitals studied. Therefore cost estimation methodologies should be incorporated in the management of these clinical services.

(Rev Med Chile 2013; 141: 1371-1381)

Key words: Costs and Costs Analysis; Economics; Health Care Costs; Intensive care.

L as organizaciones de servicios, sean éstas públicas o privadas, normalmente emplean cantidades significativas de mano de obra ${ }^{1}$. El sector salud no es ajeno a dicha realidad, dada la naturaleza del servicio y los niveles de complejidad que allí se enfrentan, el costo del recurso humano, generalmente de naturaleza indirecta, es el más significativo ${ }^{2}$.

En la determinación de los costos, los sistemas tradicionales trabajan bien con sistemas sencillos de producción, intensivos en costos directos y con una baja incidencia de los costos indirectos. No obstante, a medida que las organizaciones crecen y sus operaciones se vuelven más complejas, se hace necesario refinar sus sistemas de costeo para mantener la exactitud de los costos de los produc-

'Facultad de Ciencias
Empresariales. Universidad
de Talca.
${ }^{2}$ Unidad de Cuidados
Intensivos. Hospital Base
de Curicó.
'Unidad de Cuidados
Intensivos. Hospital
Regional de Talca.
a'Contador Público y
Auditor.
Proyecto de investigación
desarrollado entre el 01 de
diciembre de 2010 hasta
el 01 de marzo de 2012,
financiado por el Fondo
Nacional de Investigación
en Salud (FONIS).
Recibido el 16 de agosto
de 2012, aceptado el 23 de
septiembre de 2013.
Correspondencia a:
Sandra Alvear Vega.
Teléfono: $56-71$-200337,
Casilla 321. Talca.
E-mail: salvear@utalca.cl.

tos y servicios ${ }^{3}$.

Existe experiencia internacional sobre la exploración de nuevas metodologías de costos en unidad de cuidados intensivos (UCI), que permiten medir si las actividades realizadas por el personal, están agregando valor y contribuyendo al mejoramiento de los procesos ${ }^{4-5}$.

Los sistemas denominados Activity Based Costing $(\mathrm{ABC})$ se han desarrollado para medir y asignar recursos físicos que facilitan el desarrollo de actividades, colocándolas a éstas en el centro del proceso productivo de un servicio. Desde el punto de vista de la gestión hospitalaria, $\mathrm{ABC}$ permite establecer la relación causa-efecto que existe entre las actividades, así como también identificar aquellas actividades que no agregan valor ${ }^{6-7}$. 
$\mathrm{El} \mathrm{ABC}$ en primer lugar identifica las actividades, es decir, toda acción que no se puede eliminar sin afectar el valor que tiene un servicio o producto para un cliente, luego hace un seguimiento de los recursos financieros demandados por ellas, a través de la identificación de un "inductor de recurso", cuya función es reunir los costos del sistema financiero y conducirlos hacia las actividades desarrolladas. Por ejemplo, el porcentaje de tiempo de dedicación, el que normalmente se obtiene en base a entrevistas. Se finaliza con la obtención del costo total de cada una de las actividades.

En una etapa siguiente se realiza un seguimiento del costo de las actividades hasta los servicios, mediante la identificación de un "inductor de actividad", que relacionan las actividades con las demandas de los servicios individuales, por ejemplo el número de días camas. Dicho "inductor" es valorado y medido proporcionalmente por cada actividad. Finalmente, esta proporción es empleada para dirigir los costos de las actividades a los servicios o productos ${ }^{8}$.

\section{Material y Método}

Se trata de un estudio prospectivo y tiene como objetivo principal medir los costos financieros de las actividades asociadas a los tratamientos intensivos, en las Unidades de Cuidados Intensivos de adultos, UCI(s), de los hospitales públicos de Talca y Curicó, para el año 2011.

Los costos han sido medidos desde el punto de vista financiero y no desde el punto de vista económico, en tanto, se reportan costos financieros promedio y no costos marginales.

Se incluyeron todos los pacientes ingresados entre el 01 de enero y el 31 de diciembre de 2011 a las respectivas $\mathrm{UCI}(\mathrm{s})$, el criterio de inclusión fue tener como mínimo un día cama en la unidad.

Los pacientes, una vez ingresados, fueron clasificados según las siguientes patologías: sepsis, cardiovascular, respiratorias, neurológicas, traumas, digestivas, renales y otras, tomando como base el sistema de información definido por la unidad y la diferencia existente entre las prestaciones. En el grupo "otras" se incluyó post-operatorio electivo, depresión cardiorespiratoria, cetoacidosis diabética grave, entre otras.

Se aplicó el sistema ABC. Los instrumentos de medición de costos fueron validados el año 2009, en un estudio retrospectivo realizado en la UCI del Hospital de Curicó ${ }^{1}$.

Durante el mes de diciembre de 2010 se evaluó y homogenizó los sistemas de información de ambas unidades, sobre la base de instrumentos de registros y medición diseñado por los investigadores. A partir del 01 de enero de 2011, la recolección de los datos se efectuó sistemáticamente y cronológicamente por una profesional que forma parte de los respectivos equipos de trabajo de cada unidad, bajo la supervisión externa de un experto, utilizando las técnicas de análisis documental, observación directa no participativa y el análisis de los procesos.

La metodología aplicada, clasificando los costos en directos e indirectos, es la siguiente:

\section{Costos directos}

Referido a medios o factores consumidos en el proceso por un producto o servicio, o por un centro de sección de costos sobre lo que se puede calcular prácticamente su medida técnica y económica ${ }^{9}$. Los costos directos identificados en el estudio son: medicamentos, exámenes e insumos.

La cantidad de insumos médicos, exámenes e insumos, se identificaron a partir del seguimiento y registro de su consumo, en la atención de cada paciente.

\section{Costos indirectos}

Costos que incluyen el consumo de factores o medios de producción que, por afectar al proceso en su conjunto, no se pueden calcular directamente, sino por distribución?

Para asignar los costos indirectos a las actividades, desde el punto de vista metodológico, se realizaron los siguientes pasos:

1. Identificación, definición y clasificación de actividades: Para nuestro estudio las actividades fueron agrupadas en macro-actividades, es decir, varias actividades de un proceso que son afines entre sí.

Con el objetivo de elaborar un diccionario de actividades se entrevistó a cada uno de los grupos de profesionales que conforman el equipo asistencial de una UCI: directivos, médicos, enfermeras, kinesiólogos, paramédico, auxiliares. Por ejemplo, a partir de las respuestas obtenidas por cada enfermera, se procedió a validar las respuestas obtenidas por: grupo de enfermeras, enfermera supervisora y por el médico jefe de UCI, quienes realizaron un 
Tabla 1. Principales preguntas realizadas en la entrevistas a enfermeras

\begin{tabular}{|c|c|}
\hline Preguntas & Objetivo \\
\hline $\begin{array}{l}\text { ¿Puede usted describirme lo que usted hace por los pacientes de } \mathrm{UCl} \text { en un } \\
\text { día normal de trabajo? }\end{array}$ & Identificar las actividades \\
\hline $\begin{array}{l}\text { ¿Puede usted describirme lo que usted hace por los pacientes de UCl en uno } \\
\text { de los días más críticos de trabajo? }\end{array}$ & Identificar las actividades \\
\hline ¿Ciertos pacientes utilizan alguna clase de equipos? & Identificación de actividades \\
\hline $\begin{array}{l}\text { ¿Qué recursos usted emplea en sus actividades de enfermería } \\
\text { (equipos, materiales, otros)? }\end{array}$ & Identificación de recursos \\
\hline ¿Cuánto tiempo dedica usted a cada una de sus actividades? & Identificación de inductor de recurso \\
\hline ¿Cuáles son los resultados de cada actividad que usted realiza? & Identificación del inductor de actividad \\
\hline ¿Quién o qué hace uso del resultado de la actividad que usted realiza? & Identificación del objeto de costo \\
\hline
\end{tabular}

Fuente: Elaboración propia, a partir de proyecto de investigación FONIS SA10120011.

análisis exhaustivo de las actividades presentadas. El mismo procedimiento se aplicó para todos los profesionales entrevistados (Tabla 1).

2. Una vez identificada y descrita las actividades, la etapa siguiente consistió en determinar el costo de cada actividad. Las actividades consumen recursos, tales como: costo de mano de obra, electricidad, entre otros. Los costos de los recursos identificados se obtuvieron del sistema financierocontable de cada hospital.

Para efectos de distribuir el costo financiero indirectos de cada hospital a las respectivas $\mathrm{UCI}(\mathrm{s})$, se utilizó el Método Proxy ${ }^{10}$, basada en la relación existente entre los costos directos e indirectos de cada hospital en estudio.

Para distribuir dichos recursos a las actividades, se consideró el tiempo empleado en cada actividad como unidad de medida de "inductor de recursos", es decir, el tiempo necesario para desarrollar la actividad. Se solicitó a los profesionales entrevistados registrar los tiempos utilizados en cada una de las actividades identificadas, en el transcurso de un mes de trabajo. A partir de dicha información, se realizó una proyección anual.

3. Antes de asignar el costo a los pacientes, fue necesario medir la demanda que realizaban dichos pacientes de las actividades. Identificamos para cada una de ellas "inductor de actividad", es decir, una medida que nos indicara su frecuencia de realización, expresada en términos de la demanda que ejercen los pacientes sobre las actividades. Como lo son: número de hojas de evolución médico cuidados intensivos, número de hojas de enfermería, entre otros. Dicho "inductores" nos permitieron medir el número de veces de realización.

4. Finalmente, se asignó el costo desde las actividades a los pacientes. Para ello se determinaron tasas de actividad, dividiendo el costo total de cada actividad por el número total de "inductores" de cada una de ellas.

\section{Resultados}

Del total de 716 pacientes del estudio, 369 pacientes eran del hospital de Talca y 347 pacientes eran del hospital de Curicó. Noventa y ocho por ciento de ellos se encontraba egresado a la fecha de corte del estudio (Tabla 2).

La patología que presentó mayor representatividad entre los pacientes que formaron parte del estudio fueron: sepsis $27 \%(\mathrm{n}=194)$, cardiovasculares $21 \%(\mathrm{n}=149)$, trauma $18 \%(\mathrm{n}=127)$ y respiratorias $10 \%(\mathrm{n}=73)$ (Tabla 3$)$.

El elemento más distintivo en la estructura de costo del día cama fueron los costos asociados a la actividad, que incluyó costo del recurso humano y costos indirectos varios. Fluctuó entre 88 y $81 \%$ por patología en el hospital de Talca y entre 80 y 69\% por patología en el hospital de Curicó (Tabla 3).

El costo de la macro-actividad "procedimientos y terapia del paciente" fue el más relevante en términos monetario, ascendieron a \$ 140.109 en el hospital de Talca y $\$ 100.718$ en el hospital de Curicó. Le siguió en importancia el costo de las actividades asociadas a la "coordinación", equiva- 
Tabla 2. Características de la población en estudio: hospital de Talca - hospital de Curicó

\begin{tabular}{|lcc|}
\hline & Hospital de Talca & Hospital de Curicó \\
\hline Total pacientes & 369 & 347 \\
\hline Distribución por sexo & $59 \%$ Hombres & $55 \%$ Hombres \\
\hline Edad promedio & 56 años (d.e. 19) & 57 años (d.e. 19) \\
\hline Tipo de previsión & $98 \%$ Fonasa & $97 \%$ Fonasa \\
\hline Puntaje Apache promedio & 20 & 20 \\
Letalidad & $29 \%$ & $24 \%$ \\
Número de camas & 8 & 10 \\
Recurso humano & & 7 \\
Médico & 8 & 13 \\
Enfermera & 13 & 2 \\
Kinesiólogo & 4 & 13 \\
Técnico paramédico & 13 & 4 \\
Auxiliar de servicio & 6 & 1 \\
Secretaria & 1 & $1 \%$ \\
Procedencia: & & $20 \%$ \\
Clínicas privadas & $4 \%$ & $24 \%$ \\
Otros hospitales & $12 \%$ & $12 \%$ \\
Otras unidades del hospital & $25 \%$ & $42 \%$ \\
Pabellón & $27 \%$ & \\
Urgencia & $33 \%$ & \\
\hline
\end{tabular}

Fuente: Elaboración propia, a partir de proyecto de investigación FONIS SA10I20011.

lente a \$ 122.159 en el hospital de Talca y \$ 85.609 en el hospital de Curicó (Tabla 4).

A partir de las actividades, definidas en el diccionario, clasificadas en macro-actividades y por tipo de profesionales, se procedió a identificar su relevancia en términos de su valor costo en relación al conjunto de ellas.

En ambas instituciones de salud, las actividades asociadas a "procedimientos y terapia del paciente", y actividades de "coordinación", realizadas por los médicos residentes, fueron las que demandaron mayor cantidad de recursos. Las actividades asociadas a procedimientos y terapia ascendieron a \$ 76.196 en el hospital de Talca y \$ 50.971 en el hospital de Curicó, en promedio por cada día cama, sin considerar el costo adicional de exámenes, medicamentos e insumos. Las actividades de coordinación ascendieron a un costo de \$ 42.591 en el hospital de Talca y \$26.497 en el hospital de Curicó (Tabla 5).

En ambas instituciones de salud, las actividades realizadas por las enfermeras, asociadas a la coordinación y procedimientos, fueron las que demandan mayor cantidad de recursos. Las acti- vidades asociadas a la coordinación ascendieron a \$ 64.583 en el hospital de Talca y \$ 48.257 en el hospital de Curicó, siendo la actividad de coordinación de traslado de pacientes la más relevante. Las actividades de procedimientos y terapia ascendieron a \$ 32.995 y hospital de Talca y $\$ 27.472$ en el hospital de Curicó, en promedio por cada día cama (Tabla 6).

Las actividades más relevantes realizadas por los kinesiólogos, fueron aquellas asociadas a procedimientos y terapia. En el hospital de Talca, el costo de dicha actividad ascendió a $\$ 16.383$ y en el hospital de Curicó ascendió a \$2.494, en promedio por cada día cama (Tabla 7).

Las actividades más relevantes realizadas por los paramédicos, fueron aquellas asociadas a la mantención del paciente. En el hospital de Talca, el costo de dicha actividad ascendió a \$17.064 y en el hospital de Curicó ascendió a \$21.688, en promedio por cada día cama (Tabla 8).

Las actividades más relevantes realizadas por los auxiliares de servicio fueron aquellas asociadas a la mantención del paciente. En el hospital de Talca el costo de dicha actividad ascendió a \$ 12.930 
Costeo basado en actividades de tratamientos intensivos - S. Alvear et al

Tabla 3. Costo promedio día cama UCI adulto, clasificada por hospital, patología y elementos de costos

\begin{tabular}{|c|c|c|c|c|c|c|c|c|c|c|c|c|c|}
\hline \multirow[t]{2}{*}{ Patología } & \multicolumn{3}{|c|}{ n pacientes } & \multicolumn{2}{|c|}{$\begin{array}{c}\text { Costo total día } \\
\text { cama } \\
\text { (pesos, \$) }\end{array}$} & \multicolumn{2}{|c|}{$\begin{array}{l}\text { Costo de } \\
\text { actividades } \\
\text { (pesos, \$) }\end{array}$} & \multicolumn{2}{|c|}{$\begin{array}{l}\text { Costo } \\
\text { exámenes } \\
\text { (pesos, \$) }\end{array}$} & \multicolumn{2}{|c|}{$\begin{array}{c}\text { Costo } \\
\text { medicamentos } \\
(\text { pesos, \$) }\end{array}$} & \multicolumn{2}{|c|}{$\begin{array}{c}\text { Costo } \\
\text { insumos } \\
\text { (pesos, \$) }\end{array}$} \\
\hline & $\begin{array}{l}\text { Hosp. } \\
\text { Talca }\end{array}$ & $\begin{array}{l}\text { Hosp. } \\
\text { Curicó }\end{array}$ & Total & $\begin{array}{l}\text { Hosp. } \\
\text { Talca }\end{array}$ & $\begin{array}{l}\text { Hosp. } \\
\text { Curicó }\end{array}$ & $\begin{array}{l}\text { Hosp. } \\
\text { Talca }\end{array}$ & $\begin{array}{l}\text { Hosp. } \\
\text { Curicó }\end{array}$ & $\begin{array}{l}\text { Hosp. } \\
\text { Talca }\end{array}$ & $\begin{array}{l}\text { Hosp. } \\
\text { Curicó }\end{array}$ & $\begin{array}{l}\text { Hosp. } \\
\text { Talca }\end{array}$ & $\begin{array}{l}\text { Hosp. } \\
\text { Curicó }\end{array}$ & $\begin{array}{l}\text { Hosp. } \\
\text { Talca }\end{array}$ & $\begin{array}{l}\text { Hosp. } \\
\text { Curicó }\end{array}$ \\
\hline Cardiovascular & 59 & 90 & 149 & 612.147 & 310.755 & 540.112 & 247.171 & 25.142 & 28.147 & 21.534 & 11.917 & 25.359 & 23.520 \\
\hline Digestivas & 6 & 25 & 31 & 602.122 & 328.533 & 489.957 & 229.069 & 25.863 & 33.390 & 64.771 & 42.216 & 21.531 & 23.858 \\
\hline Sepsis & 97 & 97 & 194 & 541.963 & 314.460 & 443.263 & 217.601 & 25.486 & 30.361 & 48.843 & 43.990 & 24.371 & 22.508 \\
\hline Neurológicas & 18 & 29 & 47 & 510.826 & 290.616 & 452.009 & 206.495 & 20.390 & 23.730 & 22.283 & 44.490 & 16.144 & 15.901 \\
\hline Renales & 11 & 7 & 18 & 502.090 & 297.628 & 414.111 & 249.110 & 27.348 & 25.375 & 29.830 & 12.622 & 30.801 & 10.521 \\
\hline Trauma & 72 & 55 & 127 & 479.432 & 315.196 & 398.019 & 245.841 & 21.872 & 32.494 & 36.312 & 19.095 & 23.229 & 17.766 \\
\hline Respiratorias & 55 & 18 & 73 & 434.018 & 300.182 & 363.449 & 226.227 & 17.913 & 28.633 & 31.546 & 21.955 & 21.110 & 23.367 \\
\hline Otros & 51 & 26 & 77 & 583.296 & 296.010 & 510.391 & 226.381 & 22.717 & 27.782 & 27.243 & 26.262 & 22.945 & 15.585 \\
\hline
\end{tabular}

Fuente: Elaboración propia, a partir de proyecto de investigación FONIS SA10120011.

Tabla 4. Costo promedio de macro-actividad por día cama y hospital

\begin{tabular}{|c|c|c|c|}
\hline \multicolumn{2}{|l|}{ Macro - actividades } & \multicolumn{2}{|c|}{ Costo unitario promedio (pesos) } \\
\hline \multirow[t]{6}{*}{ Procedimiento y terapia del paciente } & Médico & 76.196 & 50.971 \\
\hline & Enfermeras & 32.995 & 27.472 \\
\hline & Kinesiólogo & 16.383 & 2.494 \\
\hline & Técnico paramédico & 3.355 & 5.245 \\
\hline & Auxiliar de servicio & 11.180 & 14.536 \\
\hline & Total & 140.109 & 100.718 \\
\hline \multirow{6}{*}{ Coordinación } & Médico & 42.591 & 26.497 \\
\hline & Enfermeras & 64.583 & 48.257 \\
\hline & Kinesiólogo & 2.731 & 373 \\
\hline & Técnico paramédico & 5.091 & 6.872 \\
\hline & Auxiliar de servicio & 7.163 & 3.610 \\
\hline & Total & 122.159 & 85.609 \\
\hline \multirow{3}{*}{$\begin{array}{l}\text { Respuesta a necesidades expresadas } \\
\text { por los pacientes }\end{array}$} & Médico & 7.758 & 4.206 \\
\hline & Enfermeras & 47.980 & 18.558 \\
\hline & Total & 55.738 & 22.764 \\
\hline \multirow[t]{3}{*}{ Mantención del paciente } & Técnico paramédico & 17.064 & 21.688 \\
\hline & Auxiliar de servicio & 12.930 & 7.266 \\
\hline & Total & 29.994 & 28.954 \\
\hline \multirow[t]{4}{*}{ Monitoreo del paciente } & Médico & 10.761 & 8.964 \\
\hline & Enfermeras & 4.772 & 9.091 \\
\hline & Kinesiólogo & 2.311 & 694 \\
\hline & Total & 17.844 & 18.749 \\
\hline
\end{tabular}


Tabla 5. Costo por actividad del médico residente

\begin{tabular}{|c|c|c|c|c|c|c|}
\hline \multirow[t]{2}{*}{$\begin{array}{l}\text { Macro- } \\
\text { actividades }\end{array}$} & \multirow[t]{2}{*}{ Descripción de actividades } & \multirow[t]{2}{*}{$\begin{array}{l}\text { Inductor } \\
\text { de actividad }\end{array}$} & \multicolumn{2}{|c|}{$\begin{array}{c}n \text { Inductores } \\
\text { (unidades) }\end{array}$} & \multicolumn{2}{|c|}{$\begin{array}{c}\text { Costo unitario } \\
\text { (pesos) }\end{array}$} \\
\hline & & & Talca & Curicó & Talca & Curicó \\
\hline & Entregar turnos (mañana y tarde) & n de días cama & 2.597 & 3.090 & 7.508 & 3.675 \\
\hline $\begin{array}{l}\text { Procedimiento } \\
\text { y terapia del } \\
\text { paciente }\end{array}$ & $\begin{array}{l}\text { Evaluar clínicamente a cada paciente } \\
\text { Dejar indicaciones para el día } \\
\text { Evaluar e instruir sobre procedimientos } \\
\text { de sedación de pacientes } \\
\text { Aplicar procedimiento médico } \\
\text { Hacer nuevos ingresos y/o egresos } \\
\text { Leer protocolos de atención } \\
\text { Total }\end{array}$ & $\begin{array}{l}\text { Evolución médico } \\
\text { cuidados intensivos } \\
\text { Hoja de enfermería } \\
\text { Hoja de enfermería } \\
\text { Evolución médico } \\
\text { cuidados intensivos } \\
\text { Hoja de epicrisis médica } \\
n \text { visita a pacientes }\end{array}$ & $\begin{array}{r}5.194 \\
2.966 \\
2.996 \\
\\
5.194 \\
362 \\
5.194\end{array}$ & $\begin{array}{r}2.972 \\
3.119 \\
3.119 \\
\\
2.972 \\
340 \\
2.926\end{array}$ & $\begin{array}{r}9.010 \\
7.889 \\
\\
3.944 \\
\\
4.004 \\
43.090 \\
751 \\
76.196\end{array}$ & $\begin{array}{r}9.170 \\
4.369 \\
\\
2.185 \\
\\
4.076 \\
26.720 \\
776 \\
50.971\end{array}$ \\
\hline $\begin{array}{l}\text { Monitoreo del } \\
\text { pacientes }\end{array}$ & $\begin{array}{l}\text { Visitar a cada paciente (mañana) } \\
\text { Visitar a cada paciente (tarde) } \\
\text { Manejar de alarma de motorización } \\
\text { Total }\end{array}$ & $\begin{array}{l}\mathrm{n} \text { visita a pacientes } \\
\mathrm{n} \text { visita a pacientes } \\
\mathrm{n} \text { de días cama }\end{array}$ & $\begin{array}{l}5.194 \\
5.194 \\
2.597\end{array}$ & $\begin{array}{l}2.926 \\
2.926 \\
3.090\end{array}$ & $\begin{array}{r}3.003 \\
3.754 \\
4.004 \\
10.761\end{array}$ & $\begin{array}{l}3.105 \\
3.881 \\
1.960 \\
8.964\end{array}$ \\
\hline $\begin{array}{l}\text { Dar respuesta } \\
\text { a solicitudes } \\
\text { de pacientes }\end{array}$ & $\begin{array}{l}\text { Dar entrevista a familiares } \\
\text { Entrevistar a pacientes } \\
\text { Total }\end{array}$ & $\begin{array}{l}\text { n de días cama } \\
\text { n de días cama }\end{array}$ & $\begin{array}{l}2.597 \\
2.597\end{array}$ & $\begin{array}{l}3.090 \\
3.090\end{array}$ & $\begin{array}{l}6.006 \\
1.001 \\
7.758\end{array}$ & $\begin{array}{r}2.940 \\
490 \\
4.206\end{array}$ \\
\hline Coordinación & $\begin{array}{l}\text { Asistir a reuniones de coordinación de } \\
\text { médico } \\
\text { Participar de las reuniones de la unidad } \\
\text { Total }\end{array}$ & $\begin{array}{l}\mathrm{n} \text { de pacientes } \\
\mathrm{n} \text { pacientes }\end{array}$ & $\begin{array}{l}369 \\
369\end{array}$ & $\begin{array}{l}347 \\
347\end{array}$ & $\begin{array}{l}21.137 \\
21.454 \\
42.591\end{array}$ & $\begin{array}{l}13.090 \\
13.407 \\
26.497\end{array}$ \\
\hline
\end{tabular}

Fuente: Elaboración propia, a partir de proyecto de investigación FONIS SA10120011.

Tabla 6. Costo por actividad de la enfermera

\begin{tabular}{|c|c|c|c|c|c|c|}
\hline \multirow[t]{2}{*}{$\begin{array}{l}\text { Macro- } \\
\text { actividades }\end{array}$} & \multirow[t]{2}{*}{ Descripción de actividades } & \multirow[t]{2}{*}{$\begin{array}{l}\text { Inductor } \\
\text { de actividad }\end{array}$} & \multicolumn{2}{|c|}{$\begin{array}{l}\text { n Inductores } \\
\text { (unidades) }\end{array}$} & \multicolumn{2}{|c|}{$\begin{array}{c}\text { Costo unitario } \\
\text { (pesos) }\end{array}$} \\
\hline & & & Talca & Curicó & Talca & Curicó \\
\hline $\begin{array}{l}\text { Procedimiento } \\
\text { y terapia de } \\
\text { los pacientes }\end{array}$ & $\begin{array}{l}\text { Entregar turno de enfermería } \\
\text { Controlar signos vitales normados } \\
\text { Aplicar procedimientos de enfermería } \\
\text { Realizar curaciones de catéteres } \\
\text { Instalar sonda naso entérales y } \\
\text { urinarias } \\
\text { Instalar catéteres arteriales } \\
\text { Total }\end{array}$ & $\begin{array}{l}\text { n de días cama } \\
\text { Hoja de enfermería } \\
\text { n de días cama } \\
\text { Hoja de enfermería } \\
\text { n de días cama } \\
\text { Hoja de enfermería }\end{array}$ & $\begin{array}{l}2.597 \\
2.966 \\
2.597 \\
2.966 \\
\\
2.597 \\
2.966\end{array}$ & $\begin{array}{l}3.090 \\
3.119 \\
3.090 \\
3.119 \\
\\
3.090 \\
3.119\end{array}$ & $\begin{array}{r}3.545 \\
6.208 \\
14.998 \\
2.483 \\
\\
4.090 \\
1.671 \\
32.995\end{array}$ & $\begin{array}{r}3.126 \\
7.742 \\
4.168 \\
5.161 \\
\\
5.210 \\
2.065 \\
27.472\end{array}$ \\
\hline $\begin{array}{l}\text { Monitoreo de } \\
\text { pacientes }\end{array}$ & $\begin{array}{l}\text { Acompañar la visita médica } \\
\text { Tomar muestra de exámenes (jornada } \\
\text { tarde, pacientes más inestables) } \\
\text { Manejar la alarma de monitorización } \\
\text { Total }\end{array}$ & $\begin{array}{l}\mathrm{n} \text { de visitas a pacientes } \\
\text { Valor costo de } \\
\text { exámenes }(\$) \\
\mathrm{n} \text { de días cama }\end{array}$ & $\begin{array}{r}5.194 \\
49.848 .270 \\
2.597\end{array}$ & $\begin{array}{r}2.926 \\
72.967 .045 \\
3.090\end{array}$ & $\begin{array}{l}2.045 \\
0,2131 \\
2.727 \\
4.772\end{array}$ & $\begin{array}{l}4.402 \\
0,13 \\
4.689 \\
9.091\end{array}$ \\
\hline Coordinación & $\begin{array}{l}\text { Recepcionar exámenes de laboratorio } \\
\text { Transcribir instrucciones médicas } \\
\text { Coordinar traslado de pacientes } \\
\text { Coordinar exámenes realizados fuera } \\
\text { de la unidad } \\
\text { Registrar ingreso/egresos de pacientes } \\
\text { Total }\end{array}$ & $\begin{array}{l}\text { Hojas de exámenes } \\
\text { Hoja de enfermería } \\
\text { n de pacientes } \\
\text { trasladados } \\
\text { Hoja de enfermería } \\
\text { n de pacientes }\end{array}$ & $\begin{array}{r}714 \\
2.966 \\
253 \\
\\
2.966 \\
369\end{array}$ & $\begin{array}{r}811 \\
3.119 \\
70\end{array}$ & $\begin{array}{r}14.878 \\
2.865 \\
34.379 \\
\\
2.865 \\
9.596 \\
64.583\end{array}$ & $\begin{array}{r}3.970 \\
6.194 \\
27.782 \\
\\
1.032 \\
9.279 \\
48.257\end{array}$ \\
\hline $\begin{array}{l}\text { Dar respuesta } \\
\text { a solicitudes } \\
\text { de pacientes }\end{array}$ & Educar a pacientes y familia & $\mathrm{n}$ de pacientes & 369 & 347 & 47.980 & 18.558 \\
\hline
\end{tabular}

Fuente: Elaboración propia, a partir de proyecto de investigación FONIS SA10120011. 
Costeo basado en actividades de tratamientos intensivos - S. Alvear et al

Tabla 7. Costo por actividad del kinesiólogo

\begin{tabular}{|c|c|c|c|c|c|c|}
\hline \multirow[t]{2}{*}{$\begin{array}{l}\text { Macro- } \\
\text { actividades }\end{array}$} & \multirow[t]{2}{*}{ Descripción de actividades } & \multirow[t]{2}{*}{$\begin{array}{l}\text { Inductor } \\
\text { de actividad }\end{array}$} & \multicolumn{2}{|c|}{$\begin{array}{c}\text { n Inductores } \\
\text { (unidades) }\end{array}$} & \multicolumn{2}{|c|}{$\begin{array}{l}\text { Costo unitario } \\
\text { (pesos) }\end{array}$} \\
\hline & & & Talca & Curicó & Talca & Curicó \\
\hline $\begin{array}{l}\text { Procedimiento } \\
\text { y terapia de } \\
\text { los pacientes }\end{array}$ & $\begin{array}{l}\text { Entregar turno de kinesiólogo } \\
\text { Atender integralmente a los pacientes } \\
\text { (respiratorio y motor) } \\
\text { Prevenir la atelectasia y neumonía } \\
\text { nosocomial } \\
\text { Mantener permeable la vía área } \\
\text { Realizar proceso de weaning } \\
\text { (ventilometría e índice de tobin) } \\
\text { Total }\end{array}$ & $\begin{array}{l}\text { n de días cama } \\
\text { n de días cama } \\
\text { n de días cama } \\
\text { n de días cama } \\
\text { n de días cama }\end{array}$ & $\begin{array}{l}2.597 \\
2.597 \\
2.597\end{array}$ & $\begin{array}{l}3.090 \\
3.090 \\
3.090\end{array}$ & $\begin{array}{l}1.260 \\
10.083 \\
1.680 \\
1.680 \\
1.680 \\
16.383\end{array}$ & $\begin{array}{c}- \\
1.287 \\
643 \\
188 \\
376 \\
2.494\end{array}$ \\
\hline $\begin{array}{l}\text { Monitoreo de } \\
\text { pacientes }\end{array}$ & $\begin{array}{l}\text { Visitar a cada paciente } \\
\text { Cambiar de posición al paciente } \\
\text { Total }\end{array}$ & $\begin{array}{l}\mathrm{n} \text { de visitas a pacientes } \\
\text { Hoja de enfermería }\end{array}$ & $\begin{array}{l}5.194 \\
2.966\end{array}$ & $\begin{array}{l}2.926 \\
3.119\end{array}$ & $\begin{array}{l}840 \\
1.471 \\
2.311\end{array}$ & $\begin{array}{l}398 \\
296 \\
694\end{array}$ \\
\hline Coordinación & $\begin{array}{l}\text { Registrar en ficha clínica y en hoja kinésica } \\
\text { Entregar turno kinesiólogo } \\
\text { Total }\end{array}$ & $\begin{array}{l}\text { Hoja de enfermería } \\
\text { n de días cama }\end{array}$ & $\begin{array}{l}2.966 \\
2.597\end{array}$ & $\begin{array}{c}3.119 \\
-\end{array}$ & $\begin{array}{l}1.471 \\
1.260 \\
2.731\end{array}$ & $\begin{array}{c}373 \\
- \\
373\end{array}$ \\
\hline
\end{tabular}

Fuente: Elaboración propia, a partir de proyecto de investigación FONIS SA10I20.
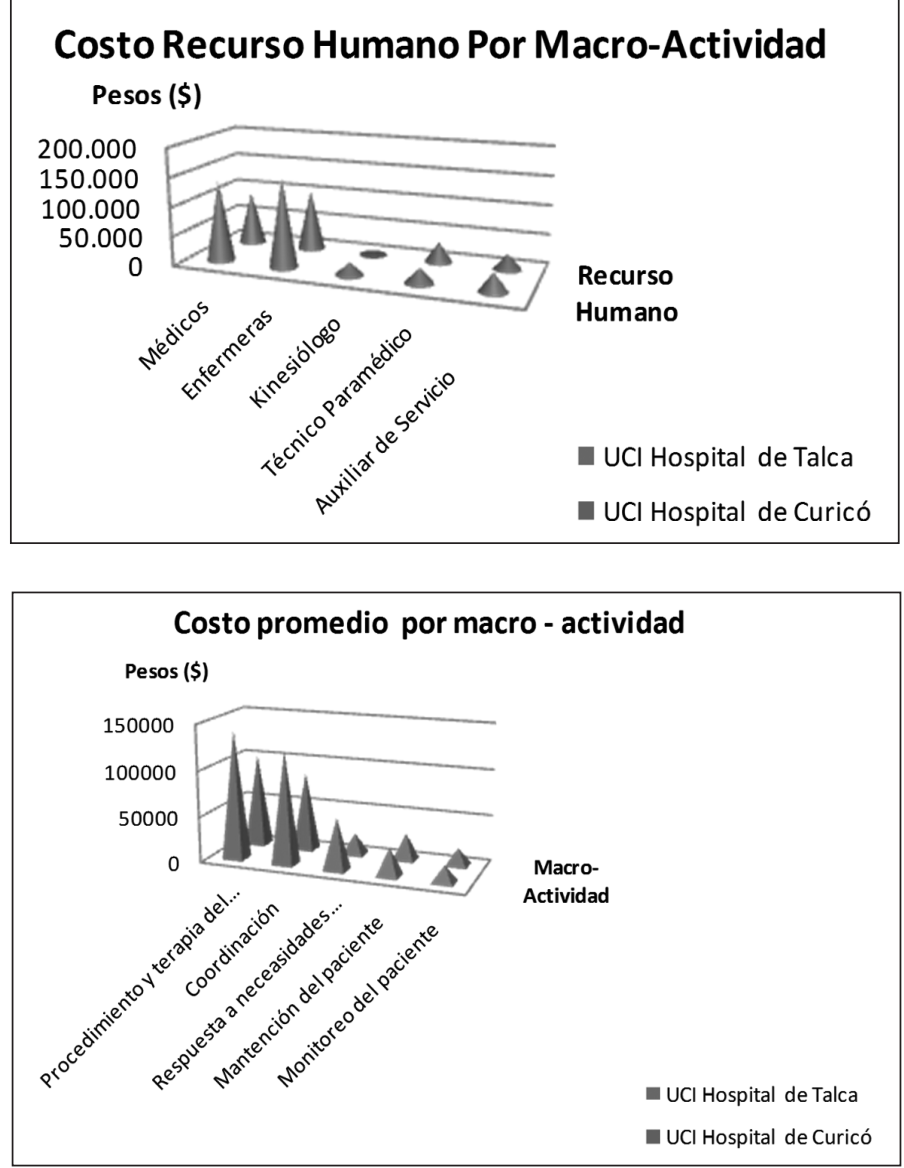

Figura 1. Participación del Recurso Humano por macro-actividad: hospital de Talca-hospital de Curicó. 
Tabla 8. Costo por actividad del técnico paramédico

\begin{tabular}{|c|c|c|c|c|c|c|}
\hline \multirow[t]{2}{*}{$\begin{array}{l}\text { Macro- } \\
\text { actividades }\end{array}$} & \multirow[t]{2}{*}{ Descripción de actividades } & \multirow[t]{2}{*}{$\begin{array}{l}\text { Inductor } \\
\text { de actividad }\end{array}$} & \multicolumn{2}{|c|}{$\begin{array}{l}\text { n Inductor } \\
\text { (unidades) }\end{array}$} & \multicolumn{2}{|c|}{$\begin{array}{c}\text { Costo unitario } \\
\text { (pesos) }\end{array}$} \\
\hline & & & Talca & Curicó & Talca & Curicó \\
\hline $\begin{array}{l}\text { Procedimiento } \\
\text { y terapia de los } \\
\text { pacientes }\end{array}$ & $\begin{array}{l}\text { Ayudar al personal de enfermería y } \\
\text { kinesiólogo } \\
\text { Preparar el carro para aplicar } \\
\text { procedimientos } \\
\text { Preparar bandeja de tratamientos } \\
\text { Controlar los signos vitales cuando } \\
\text { delega la enfermera } \\
\text { Total }\end{array}$ & $\begin{array}{l}\text { n de días cama } \\
\text { n de visitas a } \\
\text { pacientes } \\
\text { n de días cama } \\
\text { Hoja de enfermería }\end{array}$ & $\begin{array}{l}5.194 \\
2.597\end{array}$ & $\begin{array}{l}3.090 \\
2.926 \\
3.090\end{array}$ & $\begin{array}{r}674 \\
\\
225 \\
2.358 \\
\\
98 \\
3.355\end{array}$ & $\begin{array}{r}2.158 \\
1.282 \\
1.618 \\
\\
187 \\
5.245\end{array}$ \\
\hline $\begin{array}{l}\text { Mantención de } \\
\text { los pacientes }\end{array}$ & $\begin{array}{l}\text { Realizar el aseo a los pacientes } \\
\text { Administrar los alimentos a los pa- } \\
\text { cientes e hidratar a los incapacitados } \\
\text { Mantener la unidad del paciente } \\
\text { limpia y cómoda } \\
\text { Bañar en cama, aseando cavidades, } \\
\text { genitales y pelo del paciente } \\
\text { Preparar y revisar tratamiento oral e } \\
\text { inyectable y registrarlo } \\
\text { Aspirar secreciones } \\
\text { Realizar curaciones } \\
\text { Ayudar en procedimientos de } \\
\text { instalación de: catéter, sonda, } \\
\text { drenaje, línea arterial } \\
\text { Cumplir con las normas vigentes } \\
\text { en relación a las IIH } \\
\text { Total }\end{array}$ & $\begin{array}{l}\text { n de días cama } \\
\text { n de días cama } \\
\text { n de días cama } \\
\text { n de días cama } \\
\text { Consumo de } \\
\text { medicamentos (\$) } \\
\text { n de días cama } \\
\text { Hoja de enfermería } \\
\text { Evolución médico } \\
\text { cuidados intensivos } \\
\text { n de días cama }\end{array}$ & $\begin{array}{r}2.597 \\
2.597 \\
2.597 \\
2.597 \\
\\
114.161 .545 \\
2.597 \\
2.966\end{array}$ & $\begin{array}{l}3.090 \\
3.090 \\
3.090 \\
3.090\end{array}$ & $\begin{array}{l}2.358 \\
2.021 \\
3.032\end{array}$ & $\begin{array}{r}5.799 \\
1.254 \\
4.316 \\
4.316 \\
\\
0.05 \\
1.214 \\
1.202\end{array}$ \\
\hline Coordinación & $\begin{array}{l}\text { Ordenar los medicamentos } \\
\text { Despachar recetas médicas desde } \\
\text { farmacia } \\
\text { Cooperar con el médico y la } \\
\text { enfermera en el ingreso del paciente } \\
\text { Acompañar al paciente junto con la } \\
\text { enfermera, cuando éste sale de la } \\
\text { unidad } \\
\text { Registrar en ficha clínica los } \\
\text { procedimientos } \\
\text { Acompañar en la recepción y } \\
\text { entrega de turnos de enfermería } \\
\text { Total }\end{array}$ & $\begin{array}{l}\text { Consumo de } \\
\text { medicamentos }(\$) \\
\text { Consumo de } \\
\text { medicamentos }(\$) \\
\text { Hoja de enfermería } \\
\text { n de días cama } \\
\text { Hoja de enfermería } \\
\text { Hoja de enfermería }\end{array}$ & $\begin{array}{l}114.161 .545 \\
114.161 .545\end{array}$ & $\begin{array}{c}137.868 .327 \\
137.868 .327\end{array}$ & $\begin{array}{l}1.770 \\
1.278 \\
5.091\end{array}$ & $\begin{array}{l}1.618 \\
1.870 \\
1.603 \\
6.872\end{array}$ \\
\hline
\end{tabular}

Fuente: Elaboración propia, a partir de proyecto de investigación FONIS SA10I20.

y en el hospital de Curicó ascendió a \$ 7.266, en promedio por cada día cama (Tabla 9).

Todas las patologías presentaron una desviación desfavorable con respecto al arancel fijado por Fonasa, para el día cama 2011, equivalente a $\$ 192.160$.

\section{Discusión}

La aplicación del sistema de costeo basado en actividades permite dos instancias de análisis de los datos obtenidos, la primera de ella entendida como perspectiva financiera y la segunda como perspectiva operacional ${ }^{11}$. En el presente artículo, nos centraremos en la perspectiva operacional. Desde el punto de vista financiero, existen trabajos publicados recientemente ${ }^{12}$.

Desde este enfoque, la aplicación del sistema de costeo basado en actividades facilita la detección de mejoras en los métodos de trabajo de las distin- 
Tabla 9. Costo por actividad del auxiliar de servicio

\begin{tabular}{|c|c|c|c|c|c|c|}
\hline \multirow[t]{2}{*}{$\begin{array}{l}\text { Macro- } \\
\text { actividades }\end{array}$} & \multirow[t]{2}{*}{ Descripción de actividades } & \multirow[t]{2}{*}{$\begin{array}{l}\text { Inductor } \\
\text { de actividad }\end{array}$} & \multicolumn{2}{|c|}{$\begin{array}{l}\text { n Inductor } \\
\text { (unidades) }\end{array}$} & \multicolumn{2}{|c|}{$\begin{array}{l}\text { Costo unitario } \\
\text { (pesos) }\end{array}$} \\
\hline & & & Talca & Curicó & Talca & Curicó \\
\hline \multirow{3}{*}{$\begin{array}{l}\text { Procedimiento } \\
\text { y terapia de los } \\
\text { pacientes }\end{array}$} & $\begin{array}{l}\text { Tomar las radiografía de tórax o } \\
\text { abdomen y revelarlas }\end{array}$ & $\begin{array}{l}\text { n de pacientes C.V } \\
\text { y respiratorios }\end{array}$ & 114 & 108 & 10.489 & 14.327 \\
\hline & Participar en la recepción de turno & n de días cama & 2.597 & 3.090 & 691 & 209 \\
\hline & Total & & & & 11.180 & 14.536 \\
\hline \multirow[t]{10}{*}{$\begin{array}{l}\text { Mantención de } \\
\text { los pacientes }\end{array}$} & $\begin{array}{l}\text { Asear utensilios usados por el } \\
\text { paciente }\end{array}$ & n de días cama & 2.597 & 3.090 & 1.381 & 954 \\
\hline & $\begin{array}{l}\text { Realizar y mantener el aseo de la } \\
\text { unidad }\end{array}$ & n de días cama & 2.597 & 3.090 & 2.763 & 1.282 \\
\hline & $\begin{array}{l}\text { Ayudar al personal paramédico en } \\
\text { el cambio de posición del paciente }\end{array}$ & n de días cama & 2.597 & 3.090 & 652 & 447 \\
\hline & $\begin{array}{l}\text { Servir el desayuno, almuerzo y cena } \\
\text { a médico y enfermeras de turno }\end{array}$ & n de días cama & 2.597 & 3.090 & 460 & 566 \\
\hline & $\begin{array}{l}\text { Retirar los insumos de aseo: cloro, } \\
\text { líquido para piso }\end{array}$ & n de días cama & 2.597 & 3.090 & 153 & 679 \\
\hline & $\begin{array}{l}\text { Ayudar en la realización del aseo } \\
\text { del paciente }\end{array}$ & n de días cama & 2.597 & & 3.837 & - \\
\hline & $\begin{array}{l}\text { Ayudar en la realización de } \\
\text { curaciones al paciente y realización } \\
\text { de masajes }\end{array}$ & n de días cama & 2.597 & & 1.842 & - \\
\hline & $\begin{array}{l}\text { Contabilizar y retirar el vestuario y } \\
\text { ropas de cama desde lavandería }\end{array}$ & n de días cama & 2.597 & 3.090 & 1.612 & 1.842 \\
\hline & $\begin{array}{l}\text { Mantener stock de ropa limpia en } \\
\text { la unidad }\end{array}$ & n de días cama & 2.597 & 3.090 & 230 & 1.490 \\
\hline & Total & & & & 12.930 & 7.260 \\
\hline \multirow[t]{7}{*}{ Coordinación } & $\begin{array}{l}\text { Ayudar en el ingreso y traslado del } \\
\text { paciente }\end{array}$ & $\mathrm{n}$ de pacientes & 369 & 347 & 4.861 & 3.610 \\
\hline & $\begin{array}{l}\text { Llevar muestras para examen } \\
\text { a la unidad de laboratorio y retirar } \\
\text { dichos exámenes }\end{array}$ & $\begin{array}{l}\text { Valor costo de } \\
\text { exámenes }(\$)\end{array}$ & 49.848 .270 & 72.967 .045 & 0.07 & 0.04 \\
\hline & $\begin{array}{l}\text { Trasladar pedidos de farmacia, } \\
\text { bodega y economato }\end{array}$ & $\begin{array}{l}\text { Consumo de } \\
\text { medicamentos }(\$)\end{array}$ & 114.161 .545 & - & 0.02 & - \\
\hline & $\begin{array}{l}\text { Retirar informes de la unidad de } \\
\text { apoyo de diagnóstico }\end{array}$ & n de días cama & 2.597 & & 77 & \\
\hline & $\begin{array}{l}\text { Retirar el suero desde bodega y } \\
\text { cargar el suero }\end{array}$ & n de días cama & 2.597 & . & 844 & - \\
\hline & Control de visitas del paciente & n de días cama & 2.597 & - & 1.381 & \\
\hline & Total & & & & 7.163 & 3.610 \\
\hline
\end{tabular}

Fuente: Elaboración propia, a partir de proyecto de investigación FONIS SA10I2001.

tas áreas de la unidad en estudio, proporcionando información de costos acerca de las actividades y procesos asociados a las prestaciones de salud, facilitando de esta forma información para la evaluación de desempeño de las personas que participan de dichos procesos ${ }^{13-14}$.

Lo anterior, según los resultados obtenidos en el estudio, se manifiesta de la siguiente forma:

1. La macro actividad asociada a "procedimientos y terapias del paciente" es la que demanda mayor cantidad de recursos y se muestra como la actividad más relevante del proceso para todos los profesionales de la salud que participan directamente en la atención del paciente (médico, enfermeras, kinesiólogo). (Tablas 5, 6 y 7 ).

En dicho contexto, "la recepción y entrega de turno" es una actividad que reviste una particular importancia desde el punto de vista de la calidad, en una UCI, en el sentido que permite dar continuidad a la atención y tratamiento del paciente, 
en tanto, debería revisarse continuamente los procedimientos realizados.

Paralelamente, las actividades asociadas a la "mantención del paciente", desarrolladas por el personal técnico paramédico y auxiliar de servicio, son relevantes en el proceso de atención de un paciente crítico (Tablas 8 y 9).

Se puede inferir que la aplicación de $A B C$ permite tener una visión estratégica y global de la incidencia de las actividades en el costo del día cama UCI.

2. Del análisis de los datos se desprende que las actividades asociadas al "monitoreo del paciente”, son aquellas que debería demandar mayor atención, principalmente de parte del profesional médico y enfermera, en el sentido de encontrar oportunidades de mejora (Tablas 5 y 6 ).

Dicha acción es especialmente importante para los pacientes internados en las unidades de cuidados intensivos. Según estudios fenomenológicos se ha concluido que, para un paciente crítico, los elementos que forman parte del cuidado (ventilación asistida, catéter, entre otros), aún reconociendo su necesidad y utilidad, son motivos de sufrimiento y contribuyen a la dureza de la experiencia de estar críticamente enfermo ${ }^{15}$.

Se puede inferir que la aplicación de $\mathrm{ABC}$, permite analizar las actividades relevantes, que son las que proporcionan las más grandes oportunidades de mejora.

3. Las actividades asociadas a la "coordinación”, desarrolladas principalmente por médico, enfermeras y kinesiólogos, desplazan en importancia en la visión estratégica del proceso a las actividades asociadas al "monitoreo del paciente". Es importante revisar las actividades que la componen, principalmente, en relación al tiempo dedicado a reuniones internas y coordinación de acciones para el traslado de pacientes fuera de la unidad. Es importante señalar que en el hospital de Curicó se considera como paciente trasladado los que salen fuera del hospital, en cambio en el hospital de Talca se consideran también, los enviados a otras unidades del hospital (Tablas 4, 5 y 6 ).

También se observa, en ambas unidades, que el costo asociado al profesional enfermera(o) es el más relevante, $41,1 \%$ en UCI Talca y $40,3 \%$ en UCI Curicó (Figura 1). Según estudio realizado en $45 \mathrm{UCI}(\mathrm{s})$ de 10 países europeos la actividad de las enfermeras fluctúa entre 30 y $35 \%$ del costo total de la actividad de las $\mathrm{UCI}(\mathrm{s})^{16}$. Teniendo presente un referente internacional, se observa aquí un potencial de crecimiento, potenciando las buenas prácticas y desincentivando aquellas acciones que no agregan valor al paciente.

Se puede inferir que $\mathrm{ABC}$ permite identificar las actividades que no son necesarias, esto es, aquellas que no son apreciadas por los pacientes o no son esenciales para la marcha de la unidad.

4. Para comparar el desempeño entre ambas unidades, es necesario identificar una práctica más avanzada, sobre la base de estimaciones de costos. Una rigurosa disciplina de medición de los costos y de mejora de valor, es la mejor manera de impulsar el progreso del sistema ${ }^{17,18}$.

Se necesita estimar los costos de cada actividad, sobre la base de la definición de estándares de eficiencia de cantidad y precio alcanzables por cada unidad. Adicionalmente, realizar estimaciones de costos para las mismas actividades en otros sectores comparables, por ejemplo, el sector privado, lo cual requiere recurrir a fuentes externas, en busca de información confiable de costos.

Luego, a partir de los resultados obtenidos de una medición paralela, permitiría desarrollar y planificar actuaciones para igualar, mejorar y superar el modelo ${ }^{19}$.

No obstante, para efectos del estudio, la medición se realizó teniendo presente las prácticas normales de trabajo, ninguna de las unidades presentaba un desempeño superior. Sin embargo, teniendo presente lo anterior, si se compara el desempeño por UCI(s) (Talca y Curicó), se observa que existen opciones de mejoramiento para amabas unidades (Tablas 5, 6, 7, 8 y 9). La UCI del hospital de Curicó tiene una dotación de 10 camas y con un equipo de salud conformado por 40 funcionarios (en promedio 4 por cama), atendió 3.090 días camas. Paralelamente, la UCI del hospital de Talca, tiene una dotación de 8 camas y con un equipo de salud conformado por 45 funcionarios (en promedio 6 por cama) atendió 2.597 días camas.

Teniendo presente que el costo asociado al personal es de naturaleza fija en el total y variable en la unidad, es decir, frente a cambios en los niveles de producción, dicho costo puede variar, definición asociada al concepto de apalancamiento operativo ${ }^{20}$. Dicho apalancamiento es menor en el hospital de Talca, dado que su volumen de producción es menor, por ello presenta costos más altos, especialmente en las actividades asociadas a procedimiento y coordinación (Figura 2). 
Existen diferencias en la infraestructura disponible, el hospital de Talca, en relación al hospital de Curicó, producto del terremoto operaba en condiciones más desfavorables que el hospital de Curicó.

Se puede inferir que ABC permite comparar una actividad con una similar de otro hospital o de otra área del hospital.

\section{Conclusión}

El costo de las actividades, presenta diferencias significativas entre ambas UCI(s). Por ello, es necesario, la incorporación de métodos de costeos que permitan identificar las actividades que no agregan valor, facilitando la toma de decisiones de la unidad y propendiendo en el fortalecimiento de los sistemas de financiamiento.

\section{Referencias}

1. Alvear S, Canteros J, Rodríguez P. Estudio retrospectivo de costos de tratamientos intensivos por paciente y día cama. Rev Med Chile 2010; 138: 558-66. Disponible en www.scielo.cl. [Consultado el 14 de mayo de 2012].

2. Player T. Activity-Based Analyses Lead to Better Decision Making. Healthcare Financial Management; Aug 1998; 52,8; Disponible en: http://search.proquest.com. [Consultado el 14 de mayo de 2012].

3. Pines J, Fager S, Milzman DA. Review of costing methodologies in critical car estudies. Journal of Critical Care 2002; 17 (3): 181-7.

4. Edbrooke DL, Stevens VG, Hibbert CL, Mann AJ, Wilson AJ. A new method of accurately identifying costs of individual patients in intensive care: the initial results. Intensive Care Med 1997; 23: 645-50.

5. Rossi C, Simini B, Brazzi L, Rossi G, Radrizzani D, Lapichino G, et al. Variable costs of ICU patients: a multicenter prospective study. Intensive Care Med 2006; 32: 545-52.

6. Baker J, Boyd G. Activity-Based Costing in the Operating Room at Valley View Hospital. Journal of Health Care Finances; Fall 1997; 24,1; Disponible en: http:// search.proquest.com. [Consultado el 14 de mayo de 2012].

7. Mallo C, Kaplan R, Meljem S, Giménez C. Contabilidad de Costos Estratégica de Gestión. $1^{\circ}$ Edición. Madrid. Prentice Hall Iberia 2000; 359-76.

8. Cooper R, Kaplan RS. Activity-Based Systems: Measuring The Costo of Resource Usage. Accounting Horizons; 1992; 6; 3. Disponible en: http://search.proquest. com [Consultado el 14 de mayo de 2012].
9. Noel D, Padilla R. Contabilidad Administrativa. 6a Edición. México. McGraw-Hill 2002; 33-45.

10. Minsal. 2010. Informe Final. Estudio de Costo-Efectividad de Intervenciones en Salud. Ministerio de Salud. Informe Final. Disponible en: http://desal.minsal.cl/ DOCUMENTOS/PDF/GES/1.2/01CostoEfectividad.pdf [Consultado el 14 de mayo de 2012].

11. Player S. Activity-based analyses to better decisión making. Healthcare Financial Management; Aug 1998; 52, 8; Disponible en: http://search.proquest.com [Consultado el 14 de mayo de 2012].

12. Alvear S, Canteros J, Jara J, Rodríguez P. Costos reales de tratamientos intensivos por paciente y día cama. Rev Med Chile 2013; 141: 202-8. Disponible en www.scielo. cl. [Consultado el 22 de abril de 2013].

13. Shander A, Hofmann A, Ozawa S, Theusinger O, Gombatz H, Spahn D. Activity-based costs of blood transfusions in surgical patients at four hospitals. Transfusion 2010; 50 (4): 753-65, 13p, 5 Charts, 3 Graphs. Disponible: http://web.ebscohost.com. [Consultado el 14 de junio de 2012].

14. Yen-Lu Lin B, Chao T, Yao Y, Tu Sh, Wu Ch, Chern I, et al. How Can Activity-Based Costing Methodology Be Performed as a Powerful Tool to Calculate Costs and Secure Appropriate Patient Care? Journal of Medical Systems 2007; 31 (2): 85-90, 6p, 1 Diagram, 3 Charts. Disponible: http://web.ebscohost.com [Consultado el 14 de junio de 2012].

15. Beltrán-Salazar OA. Aquichan 2009; 9 (1): 23-37, 15p. La experiencia de estar hospitalizado en una unidad de cuidado intensivo). Disponible: http://web.ebscohost. com.

16. Reis Miranda D, Jegers M. Monitoring costs in the ICU: a search for a pertinent methodology. Acta Anaesthesiol Scand 2012; 56: 1104-13.

17. Porter M. What Is Value in Health Care? The New England Journal of Medicine 2010; 363: 23. Disponible en: http://www.nejm.org/doi/full/10.1056/NEJMp1011024.

18. Lee T. Putting the Value Framework to Work. The New England Journal of Medicine (2010); 363; 23. Disponible en: http://www.nejm.org/doi/full/10.1056/ NEJMp1011024.

19. Tan S, Bakker I, Hoogendoorn M, Kapila A, Martin J, Pezzi A, et al. Direct Cost Analysis of Intensive Care Unit Stay in Four European Countries: Applying a Standardized Costing Methodology. Value in Health (Elsevier Science) 2012; 15 (1): 81-6, 6p. Disponible: http://web.ebscohost.com [Consultado el 14 de junio de 2012].

20. Hansen D, Mowen M. Administración de Costos. $3^{\text {a }}$ Edición. México. Thomson Editores, S.A. 2003; 724-5. 